\title{
Allozyme variation in Louisiana irises: a test for introgression and hybrid speciation
}

\author{
M. L. Arnold, ${ }^{*} \ddagger$ \\ J. L. Hamrick* ${ }^{*}$ and \\ B. D. Bennett*
}

Departments of $*$ Genetics and $†$ Botany, University of Georgia, Athens, Georgia, 30602, U.S.A.

Genetic variation present in 599 individual Iris samples was examined at 50 presumptive loci (isozymes). The samples included allopatric populations of $I$. fulva, I. hexagona, $I$. brevicaulis and the putative hybrid species, $I$. nelsonii. In addition, we examined two populations from areas of overlap involving $I$. fulva with $I$. hexagona, and $I$. fulva with $I$. brevicaulis and $I$. hexagona. Allozyme data for both of these hybrid populations suggest the presence of genes from $I$. fulva, $I$. brevicaulis and $I$. hexagona. Bidirectional introgressive hybridization is indicated between $I$. fulva and $I$. hexagona. Individuals from a parapatric association between these two species possess various combinations of $I$. fulva and $I$. hexagona diagnostic markers. The pattern of genetic variation in this sample indicates the presence of advanced hybrid generations and localized introgression; this finding is in accord with a previous molecular genetic analysis (Arnold et al., 1990). Furthermore, currently allopatric populations of I. fulva and I. hexagona were found to have low frequencies of marker alleles diagnostic for the alternate species. These populations also contain a low frequency of alternate, diagnostic ribosomal DNA repeat length variants (Arnold et al., 1990). The allozyme (and molecular) data suggest that these allopatric samples represent previously introgressed populations. Findings from the allozyme survey indicate that $I$. nelsonii contains an array of markers characteristic for $I$. fulva, $I$. hexagona and $I$. brevicaulis. Furthermore, some of these markers are found in a present-day hybrid population involving $I$. fulva, $I$. hexagona and $I$. brevicaulis. These findings suggest that $I$. nelsonii is of hybrid origin, deriving from hybridization between $I$. fulva, I. hexagona and $I$. brevicaulis. The genetic analysis along with previous chromosomal, distributional and demographic studies of the Louisiana Iris species suggests that a number of mechanisms may have been involved in the stabilization of this hybrid derivative.

\section{INTRODUCTION}

Natural hybridization has been inferred for numerous plant and animal species complexes (e.g., Helianthus, Heiser et al., 1969; Eucalyptus, Potts and Reid, 1988; Iris, Anderson, 1949; Caledia, Moran and Shaw, 1977; Marchant et al., 1988; Geomys, Baker et al., 1989; Menippe, Bert and Harrison, 1988). In addition to describing the occurrence of hybridization, a number of the more detailed studies of natural hybridization between animal taxa have detected the transfer of genetic material between the hybridizing forms (i.e., introgressive hybridization or introgression; Anderson and Hubricht, 1938). In plant systems, however, there have been only a limited number of genetic analyses of natural hybridization (Soltis, 1985; Rieseberg et al., 1988; Doyle and Doyle,

$\ddagger$ To whom correspondence should be addressed.
1988; Arnold et al., 1990; dePamphilis and Wyatt, 1990; Rieseberg et al., 1990). When such studies have been undertaken, a variety of evolutionary patterns have been discovered. Thus, data from genetic studies of plant hybridization indicate (1) hybridization with little or no apparent introgression (Doyle and Doyle, 1988), (2) hybridization with introgression occurring only in sympatric, localized associations (Rieseberg et al., 1988), (3) hybridization with introgression apparent in parapatric and allopatric populations of the hybridizing species (Arnold et al., 1990; dePamphilis and Wyatt, 1990) and (4) hybridization that has resulted in the origin of a new species (Rieseberg et al., 1990). It is significant to note that genetic analyses have varied with regard to their concordance with morphological data from the same hybridization events. Thus, dePamphilis and Wyatt (1990) supported the conclusions drawn from previous morphological analyses of Aesculus 
(Hardin, 1957 $a, b)$. Likewise, Doyle and Doyle (1988) found agreement between morphological, pollen stainability and molecular genetic data for Claytonia species and hybrids. In contrast, Rieseberg et al. (1988) rejected an earlier conclusion concerning the introgression-mediated origin of a plant race (Heiser, 1949). Data from the genetic analysis of Rieseberg et al. (1990) supported one instance of recombinational (hybrid) speciation while rejecting a second putative example. Finally, molecular genetic analyses of the "Louisiana Iris" species (Arnold et al., 1990) have supported the original finding of introgressive hybridization (Anderson, 1949), rather than the conclusions of Randolph et al. (1967) who argued that introgression had not occurred between these species. The lack of concordance between morphological and genetic data may reflect a lower sensitivity of morphological analyses in detecting the aftermath of natural hybridization. In this regard, Anderson and Hubricht (1938) concluded that the introduction of a small amount of genetic material from one taxon, into another, might be difficult, if not impossible, to detect using morphological characters.

One of the examples given above involved species belonging to the genus Iris. Morphological variation demonstrated by Iris fulva, I. hexagona (=I. hexagona var. giganti-caerulea; Anderson, 1949) and their natural hybrids (Riley, 1938) was used by Anderson (1949) as a "typical example" of the process of introgressive hybridization. In contrast, Randolph et al. (1967), after examining morphological characters and marker chromosomes in "sympatric" and "allopatric" populations of I. fulva, I. hexagona (=I. giganticaerulea; Randolph et al., 1967) and a third species, I. brevicaulis, concluded that there was no evidence that introgression had taken place in this species complex. This conclusion was based on the absence of marker chromosomes in allopatric populations of the two species and the inability of the authors to detect changes in populational means or variances for 13 morphological characters in allopatric populations. Their analyses did, however, lead Randolph et al. (1967) to conclude that a new species ( $I$. nelsonii) had originated as a result of hybridization between I. fulva, I. hexagona and, possibly, I. brevicaulis (Randolph, 1966).

I. fulva and $I$. hexagona come into contact in southern Louisiana when bayou systems derived from the Mississippi river end in fresh water marshes and swamps. I. fulva occurs in the understory environment associated with bayou margins while $I$. hexagona inhabits open freshwater swamps (Viosca, 1935; Bennett, 1989). These two species differ in chromosome number and apparent structural rearrangements ( $I$. fulva, $2 N=42 ; I$. hexagona, $2 N=44$ ) and natural hybrids demonstrate lower pollen fertilities relative to natural populations of the parental species (Randolph et $a l ., 1967)$. In spite of the partial reproductive isolation between $I$. fulva and I. hexagona, almost synchronous flowering times and the sharing of common pollinators results in the production of natural hybrids in areas of overlap (Anderson, 1949; Randolph et al., 1967; Bennett, 1989; Arnold et al., 1990). In comparison to the former two species, $I$. brevicaulis ( $=I$. foliosa; Viosca, 1935) occurs in much drier habitats (unimproved pastures and hardwood forests; Viosca, 1935; Randolph et al., 1967). This species shares the same diploid number of chromosomes as I. hexagona (i.e., 44;), but possesses a unique set of marker chromosomes (Randolph et al., 1961). Although there is an asynchrony in the flowering times of $I$. brevicaulis and the other two species, natural hybrid populations between I. fulva and I. brevicaulis have been reported (Randolph et al., 1967).

The present examination of isozyme variation represents an extension of an earlier genetic analysis of I. fulva, I. hexagona and natural hybrid populations (Arnold et al., 1990). In the previous analysis, molecular markers (i.e., ribosomal DNA repeat length) were examined for allopatric populations of I. fulva and I. hexagona, and a parapatric association of these two species. The results supported the occurrence of introgressive hybridization between I. fulva and I. hexagona, both in the parapatric association and into allopatric populations of both species. These findings agree with Anderson's (1949) original use of these two species as the paradigm for introgressive hybridization, but are in disagreement with the conclusions of Randolph et al. (1967). The present analysis of isozyme variation includes most of the populations of $I$. fulva, $I$. hexagona and hybrids examined in the molecular study. In addition, we examined a single population of $I$. brevicaulis, a sample of the putative hybrid species, $I$. nelsonii and an area of apparent overlap between I. fulva, I. hexagona and I. brevicaulis. The isozyme analysis addressed the following questions: (1) Do additional genetic data (i.e., isozyme variation) support or reject the hypothesis that introgression has occurred between I. fulva and I. hexagona?; (2) If introgression is occurring, are isozyme and rDNA data concordant with respect to the direction of introgression?; (3) Is the genetic composition of the I. fulva $\times I$. 
hexagona hybrid population the same, with respect to the distribution of recombinant and parental types, based upon the molecular and biochemical data sets?; (4) Does the area of overlap between I. fulva, I. hexagona and I. brevicaulis include genetic variation indicative of hybridization?; (5) Do the genetic data support the contention that $I$. nelsonii is a hybrid derivative of $I$. fulva, $I$. hexagona and I. brevicaulis?

\section{MATERIAL AND METHODS}

Four allopatric populations of I. fulva, five allopatric populations of $I$. hexagona, one allopatric population of $I$. brevicaulis and one allopatric population of $I$. nelsonii were assayed in the isozyme analysis. One of the allopatric populations of $I$. fulva ("Kraemer" population) possessed isozyme variants that were otherwise diagnostic for I. hexagona or present in presentday hybrid populations. Similarly, one of the allopatric populations of $I$. hexagona ("Marmande" population) demonstrated isozyme variants that were otherwise only present in I. fulva and known hybrid populations. Thus, the Kraemer and Marmande populations were analysed separately from populations of their respective species when calculating allelic variation and genetic distance measurements. Two hybrid populations ("Bayou L'ourse" and "Foti" populations) were also examined for isozyme variation. The following populations were sampled for isozyme variation ( $n$ indicates the sample size for each population surveyed): I. fulva; Louisiana, East Baton Rouge Parish, Bayou Paul Rd, $n=48$; Assumption Parish, $6.92 \mathrm{~km}$ W of junction of HWY 398 and HWY 1 on HWY 398, $n=48$; Terrebonne Parish, $7.56 \mathrm{~km}$ W of junction of HWY 20 and HWY 311 on HWY 311, $n=48$; Lafourche Parish, $6.75 \mathrm{~km} \mathrm{~S}$ of Kraemer on HWY 307, $n=48$, "Kraemer" population; I. hexagona; Louisiana, St. Martin Parish, $11 \mathrm{~km} \mathrm{~N}$ of Morgan City on HWY 70, $n=$ 14; Terrebonne Parish, $11 \mathrm{~km} \mathrm{~S}$ of Houma, $n=48$, "Marmande" population; Florida, Taylor County, $10 \mathrm{~km}$ from junction of Hickory Mound Rd and HWY 98 on Hickory Mound Rd, $n=16 ; 15 \mathrm{~km}$ from junction of Hickory Mound Rd and HWY 98 on Hickory Mound Rd, $n=48$; Levy County, $13 \mathrm{~km} \mathrm{~N}$ of junction of HWY 19/98 and Hwy 24 on HWY 19/98, $n=48$; I. brevicaulis; Louisiana, St. Martins Parish, St. Martinsville, $n=48$; $I$. nelsonii; Louisiana, Vermilion Parish, $0.3 \mathrm{~km}$ from junction of HWY 330 and P-2-7 on HWY 330;
Hybrid Populations; Louisiana, Assumption Parish, $1 \mathrm{~km} \mathrm{~S}$ of junction of HWY 662 and HWY 398 on HWY 662, $n=42$, "Bayou L'ourse" population; St. Martin Parish, 108 S. Main St., St. Martinsville, $n=48$, "Foti" population.

For each of the populations sampled, leaf material was taken from individuals derived from separate rhizomes. The leaf material was either placed directly into liquid nitrogen or placed onto wet ice until it was possible to "snap-freeze" the tissue in liquid nitrogen. For long-term storage, the snap-frozen leaf material was placed at $-80^{\circ} \mathrm{C}$.

Enzyme extraction was accomplished by grinding part of a leaf under liquid nitrogen with mortar and pestle. The resulting powder was mixed with a potassium phosphate buffer (Mitton et al., 1979) and the extract was absorbed onto filter paper wicks. Wicks were stored at $-60^{\circ} \mathrm{C}$ until needed for analysis. Electrophoresis was performed using 11 per cent starch gels. Gels were stained for 19 enzyme systems: acid phosphatase (ACPH); alcohol dehydrogenase (ADH); aldolase (ALD); adenylate kinase $(\mathrm{AK})$; colorimetric esterase (CE); diaphorase (DIA); fluorescent esterase (FE); fructose-1,6-diphosphatase (F16DP); glutamate oxaloacetate transaminase (GOT); isocitrate dehydrogenase (IDH); leucine aminopeptidase (LAP); malate dehydrogenase (MDH); menadione reductase (MNR); mannose-6-phosphate isomerase (MPI); phosphoglucoisomerase (PGI); phosphoglucomutase (PGM); 6-phosphogluconate dehydrogenase (6PGDH); shikimate dehydrogenase (SKDH); and triosephosphate isomerase (TPI). Staining schedules followed Soltis et al. (1983).

Controlled crosses to confirm the genetic basis of variable banding patterns were not made. However, for all the enzyme systems used in our analysis of hybridization, the number of independent banding zones, as well as the banding patterns within each variable zone, were consistent with known enzyme substructure and compartmentalization (Gottlieb, 1981). Furthermore, an analysis of open-pollinated family arrays were entirely consistent with a genetic interpretation for all of the diagnostic loci (Hamrick and Arnold, unpublished data).

Allele frequencies were calculated for each of the polymorphic loci. The genetic similarity of each of the taxa and the presumptive hybrid populations were compared by Nei's (1972) genetic identity (I) statistic. To ease comparisons, the several populations representing I. fulva and I. hexagona were pooled for the analysis. 
Table 1 Nei's Genetic Identity values for comparisons of I. fulva (I.F.), I. nelsonii (1.N.), I. brevicaulis (I.B.), I. hexagona (I.H.), two hybrid populations (B.L. = Bayou L'ourse and Foti), one allopatric population of I. fulva (Kraemer) and one allopatric population of I. hexagona (Marmande)

\begin{tabular}{|c|c|c|c|c|c|c|c|c|}
\hline & I.F. & I.N. & I.B. & I.H. & B.L. & Foti & Kraemer & Marmande \\
\hline I.F. & - & 0.95 & 0.82 & 0.75 & 0.84 & $0 \cdot 86$ & 0.98 & 0.80 \\
\hline I.N. & & - & 0.82 & 0.71 & 0.80 & 0.86 & 0.92 & 0.76 \\
\hline I. B. & & & - & 0.77 & 0.84 & 0.90 & 0.81 & 0.81 \\
\hline I.H. & & & & - & $0 \cdot 88$ & 0.77 & 0.73 & 0.88 \\
\hline B.L. & & & & & - & $0 \cdot 86$ & 0.83 & 0.97 \\
\hline Foti & & & & & & - & 0.85 & 0.85 \\
\hline Kraemer & & & & & & & - & 0.78 \\
\hline
\end{tabular}

\section{RESULTS}

Isozyme data from the 50 presumptive loci were used to calculate Nei's $I$ values (Nei, 1972) for $I$. fulva, I. nelsonii, I. brevicaulis, I. hexagona, the Bayou L'ourse hybrid population, the Foti hybrid population, an allopatric population of I. fulva (Kraemer population) and an allopatric population of I. hexagona (Marmande population; table 1). Isozyme data for the three I. fulva populations and the four I. hexagona populations were pooled for this analysis; the average identity (I) values for comparisons within I. fulva and I. hexagona were 0.98 and 0.86 , respectively. It is not clear why the $I$ values found in the $I$. hexagona comparisons are significantly lower than for the $I$. fulva comparisons. However, it is possible that I. hexagona represents a species "complex" rather than a single species (N. Henderson, personal communication). Experimental crosses are currently underway to define levels of reproductive isolation between populations of " $I$. hexagona". The five highest $I$ values were produced in comparisons of I. fulva with the Kraemer population $(I=0.98)$, the Bayou L'ourse hybrid population with the Marmande population $(I=0.97)$, I. fulva with $I$. nelsonii $(I=$
$0.95), I$. nelsonii with the Kraemer population $(I=$ $0.92)$ and $I$. brevicaulis with the Foti hybrid population $(I=0 \cdot 90)$. The next highest $I$ values resulted from comparisons of $I$. hexagona populations with the Bayou L'ourse hybrid population and the Marmande population $(I=0 \cdot 88)$.

Four isozyme loci ( $A k-2$, Got-1, Got-2 and $P g i$-3) were found to contain "marker" alleles for I. fulva and I. hexagona (table 2). The I. nelsonii sample is fixed for those alleles characteristic for I. fulva, except at the Got-1 locus. At this locus, I. nelsonii has a predominance of the allele found in I. hexagona populations. Similarly, the Kraemer population is characterized by having alleles typical for I. fulva. The Marmande sample has allele frequencies for $A k-2$, Got-2 and Pgi-3 like those of an I. hexagona population, however, this sample contains a relatively high frequency of the I. fulva variant at Got-1. The Bayou L'ourse sample is more similar to $I$. hexagona with respect to the frequency of the marker alleles. However, this population also contains a higher frequency of the markers characteristic of $I$. fulva than does any $I$. hexagona population (fig. 1). Thirty-nine of the Bayou L'ourse individuals examined for the allozyme analysis were also assayed for ribosomal

Table 2 Frequency of marker alleles for I. fulva (I.F.), I. hexagona (I.H.), I. nelsonii (I.N.), the Bayou L'ourse hybrid sample (B.L.), an allopatric population of I. fulva (Kraemer) and an allopatric population of I. hexagona (Marmande)

\begin{tabular}{llllllll}
\hline Isozyme & Allele & I.F. & B.L. & I.H. & I.N. & Kraemer & Marmande \\
\cline { 2 - 7 }$A k-2$ & 3 & 0.00 & 0.92 & 1.00 & 0.00 & 0.00 & 1.00 \\
A $k-2$ & 4 & 1.00 & 0.08 & 0.00 & 1.00 & 1.00 & 0.00 \\
Got -1 & 3 & 0.99 & 0.12 & 0.00 & 0.30 & 1.00 & 0.39 \\
Got -1 & 4 & 0.01 & 0.88 & 1.00 & 0.70 & 0.00 & 0.61 \\
Got -2 & 3 & 0.01 & 0.82 & 0.97 & 0.00 & 0.00 & 1.00 \\
Got -2 & 4 & 0.99 & 0.18 & 0.03 & 1.00 & 1.00 & 0.00 \\
Pgi-3 & 3 & 1.00 & 0.21 & 0.04 & 1.00 & 1.00 & 0.00 \\
Pgi-3 & 5 & 0.00 & 0.79 & 0.63 & 0.00 & 0.00 & 1.00 \\
Pgi-3 & 6 & 0.00 & 0.00 & 0.33 & 0.00 & 0.00 & 0.00 \\
\hline
\end{tabular}



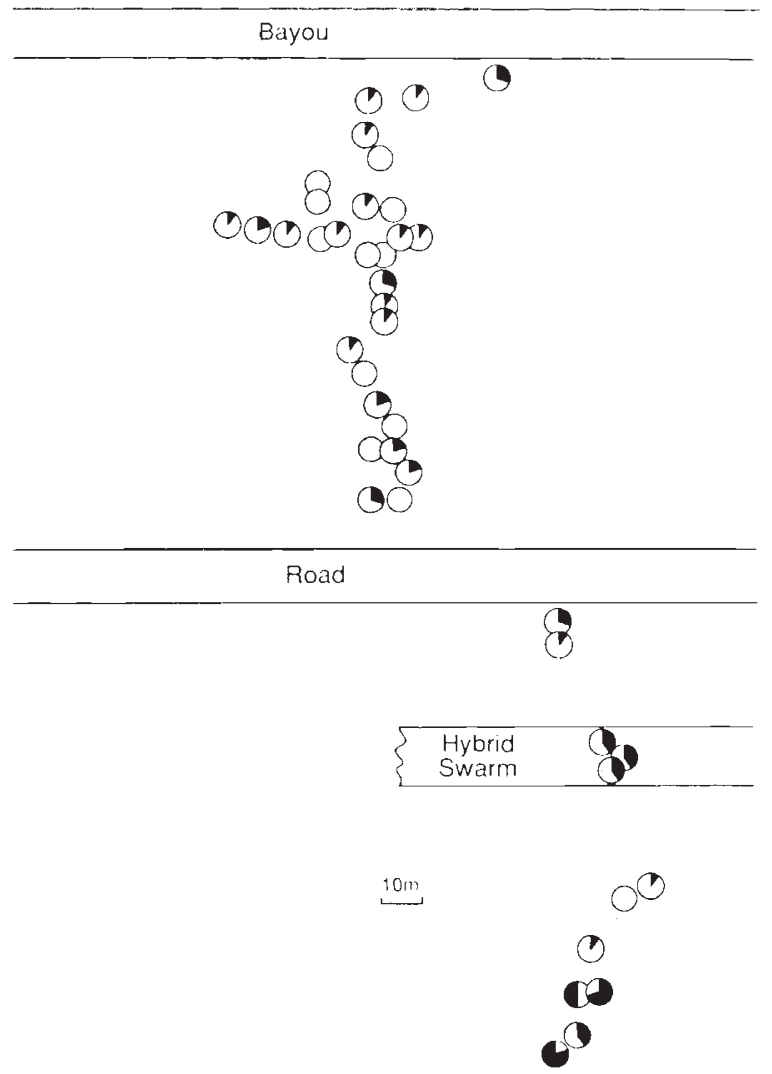

Figure 1 Relative proportion of $I$. hexagona and I. fulva genetic markers (i.e., isozyme and rDNA) present in 42 individuals sampled from the Bayou L'ourse population. The shaded portion of each pie diagram represents the relative proportion of the $I$. fulva markers. The unshaded region of each diagram represents the relative proportion of the I. hexagona genetic markers. The "Hybrid Swarm" is an area of extreme variation in floral coloration.

DNA (rDNA) variation (Arnold et al., 1990). Thus, we have included the rDNA in determining the relative proportion of $I$. fulva to $I$. hexagona genetic material for these 39 individuals (fig. 1). This was accomplished by scoring the rDNA results as "I. fulva", "I. hexagona" or "heterozygous". This analysis differs from the Arnold et al. (1990) study where these authors reported the relative amount of the two rDNA markers in each individual.

During the collection of the Foti population samples, we observed plants that possessed typical I. brevicaulis floral phenotypes (Viosca, 1935). In addition, we collected leaf material from plants that possesed vegetative and seed pod characteristics that were similar to I. fulva and I. hexagona. The Foti population contains allele frequencies that are similar to those found in I. fulva, I. brevicaulis and/or I. hexagona (table 3). Allele frequencies at $A k-2$ and $P g i-3$ demonstrate the presence of $I$. hexagona alleles. The Foti population contains significant frequencies of alleles characteristic for I. fulva at the Got-1 and Got-2 loci. Finally, I. brevicaulis alleles for the Got-2, $\mathrm{Pgi}-3$ and $\mathrm{Fe}-3$ loci are apparent in this population (table 3 ). Fig. 2 illustrates the relative position of the individual plants sampled from the Foti population, and the presence or absence of $I$. fulva, $I$. brevicaulis and I. hexagona alleles.

In addition to the marker alleles discussed above, there are several low frequency alleles shared by I. fulva, I. hexagona, I. brevicaulis or $I$. nelsonii and the Bayou L'ourse, Foti, Kraemer and/or Marmande populations (table 4).

\section{DISCUSSION}

Anderson and Hubricht (1938) and Anderson (1949) have argued that the most common outcome of the formation of "hybrid swarms" (i.e., hybrid populations containing a variety of hybrid generations) is the transfer of genes from one of the hybridizing types to the other. This expectation must now be qualified by the observation that not all genetic markers demonstrate the same propensity to cross reproductive barriers between hybridizing taxa (e.g., see Harrison, 1986; Arnold et al., 1987; Marchant et al., 1988). The "semipermeable" nature of the boundary between hybridizing taxa (Harrison, 1986) is best explained

Table 3 Frequency of I. fulva, I. hexagona and I. brevicaulis (i.e., I.F., I.H. and I.B., respectively) marker alleles for the Foti population

\begin{tabular}{llllll}
\hline Isozyme & Allele & I.F. & I.H. & I.B. & Foti \\
\hline Ak-2 & 3 & 0.00 & 1.00 & 0.00 & 0.02 \\
A $k-2$ & 4 & 1.00 & 0.00 & 1.00 & 0.98 \\
Got -1 & 3 & 0.99 & 0.00 & 0.05 & 0.49 \\
Got -1 & 4 & 0.01 & 1.00 & 0.95 & 0.51 \\
Got-2 & 1 & 0.00 & 0.00 & 0.01 & 0.09 \\
Got-2 & 2 & 0.00 & 0.00 & 0.13 & 0.10 \\
Got-2 & 3 & 0.01 & 0.97 & 0.86 & 0.53 \\
Got-2 & 4 & 0.99 & 0.03 & 0.00 & 0.27 \\
Pgi-3 & 1 & 0.00 & 0.00 & 0.01 & 0.34 \\
Pgi-3 & 3 & 1.00 & 0.04 & 0.20 & 0.34 \\
Pgi-3 & 4 & 0.00 & 0.00 & 0.50 & 0.01 \\
Pgi-3 & 5 & 0.00 & 0.63 & 0.29 & 0.26 \\
Pgi-3 & 6 & 0.00 & 0.33 & 0.00 & 0.04 \\
Fe-3 & 1 & 0.00 & 0.00 & 0.02 & 0.00 \\
Fe-3 & 2 & 0.00 & 0.00 & 0.98 & 0.63 \\
Fe-3 & 3 & 0.99 & 0.93 & 0.00 & 0.37 \\
Fe-3 & 5 & 0.01 & 0.07 & 0.00 & 0.00 \\
\hline
\end{tabular}




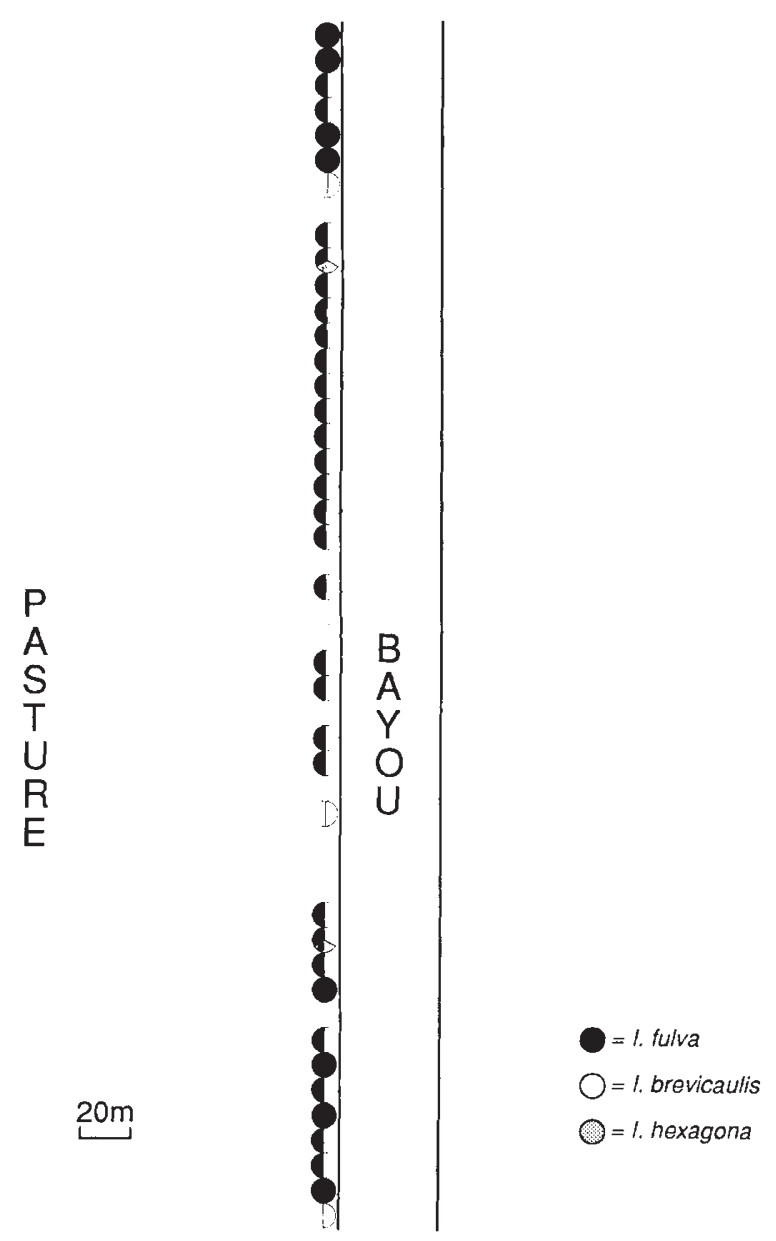

Figure 2 The distribution of I. fulva (shaded portion), I. brevicaulis (unshaded portion) and I. hexagona (stippled portion) alleles in 48 individuals from the Foti population. Note: the relative area that is shaded, unshaded and/or stippled in each of the individual diagrams does not indicate the proportion, but rather, the presence or absence of diagnostic markers for the three species.

by the interaction of natural selection, mating behaviour, historical components of the hybridization event, ecological determinants, the genetics of the markers under study, stochastic processes and/or the presence of molecular mechanisms that are capable of increasing the rate and determining the direction of the transfer of certain genomic components (Shaw et al., 1980; Marchant et al., 1988; Arnold et al., 1988; Baker et al., 1989; Rand and Harrison, 1989; Arnold et al., 1990). To test the relative importance of these factors on the outcome of hybridization, it is most useful to examine multiple genetic markers for the populations and to compare the distribution of these markers to such things as the ecological context of the hybridizing species, mating systems and modes of reproduction (i.e., sexual and asexual).

\section{Introgressive hybridization in Louisiana irises: I. fulva $\times I$. hexagona.}

The isozyme analysis of I. fulva and I. hexagona included several populations already assayed for rDNA variation. We also analysed two areas of overlap between various combinations of these species and allopatric populations of I. fulva and I. hexagona that contain diagnostic molecular markers (i.e., rDNA) from the alternate.species (Arnold et al., 1990). As with the molecular survey, the allozyme data detected a number of diagnostic alleles that were only found in allopatric populations of either I. fulva or I. hexagona. The Kraemer and Marmande populations, however, represent allopatric populations of I. fulva (Kraemer) and I. hexagona (Marmande), yet contain alleles that are considered diagnostic for the opposite species. The Marmande population also possesses alleles that are otherwise restricted to $I$. brevicaulis, although there are no known populations of $I$. brevicaulis in this parish (N. Henderson, personal communication). The presence of these alleles may be due to the retention of ancestral variation or, alternatively, may result from previous introgressive hybridization. The selection of one hypothesis over the other is enhanced if there is concordance between independent genetic data sets for the same individuals. For example, Marchant et al. (1988) were able to strongly infer the presence of geographically extensive, introgressive hybridization between two subspecies of the grasshopper Caledia captiva because of the presence of multiple, alternate molecular (mitochondrial and ribosomal DNA) and biochemical (allozyme) markers. In the present case, Arnold et al. (1990) have already reported a low frequency of the rDNA markers characterististic of $I$. hexagona in the Kraemer population and of $I$. fulva in the Marmande population. It is significant to note that an analysis of the Marmande population found it to be typical for $I$. hexagona with respect to morphological variation and aspects of clonal growth (Bennett, 1989). Thus, the Kraemer and Marmande populations are characteristic for their respective species, except that they possess low frequencies of genetic markers that are characteristic for the alternate species. Therefore, the genetic (Arnold et al., 1990; the present study) and morphological (Bennett, 1989) data for the Kraemer and Marmande populations suggest that 
Table 4 Distribution of species specific markers in I. nelsonii, Bayou L'ourse, Foti, Kraemer and Marmande populations. Values in each column represent the number of species specific alleles present. I.F. =I. fulva, I.H. =I. hexagona, I.B. $=$ I. brevicaulis, I.N. =I. nelsonii, B.L. = Bayou L'ourse. Numbers in parenthesis, adjacent to species designations, represent the numbers of unique marker alleles present in populations of that species. When a population is compared with itself, the number in the column represents the number of unique alleles characteristic for that population

\begin{tabular}{llllll}
\hline & I.N. & B.L. & Foti & Kraemer & Marmande \\
\hline I.F. (5) & 1 & 1 & 0 & 1 & 1 \\
I.H. (37) & 2 & 6 & 8 & 4 & 5 \\
I.B. (10) & 3 & 3 & 6 & 0 & 3 \\
I.N. (3) & - & - & - & - & - \\
B.L. & - & 1 & - & - & - \\
Foti & - & - & 9 & - & - \\
Kraemer & - & - & - & 3 & 3 \\
Marmande & - & - & - & - & \\
\hline
\end{tabular}

bi-directional, introgressive hybridization has occurred between I. fulva and I. hexagona. The allozyme data also suggest differential introgression of the various genetic markers. Thus, the Marmande population contains a relatively high frequency of the I. fulva diagnostic Got-1 allele and several of the low frequency alleles only present in I. fulva. In contrast, the Kraemer population is fixed for the I. fulva variant at each of the diagnostic loci (table 2), yet contains a number of alleles at other loci that are found at low frequencies only in $I$. hexagona (table 4).

In addition to the presence of alternate allozymes (and molecular markers) in the Kraemer and Marmande populations, we have also detected apparent localized introgression between $I$. fulva and $I$. hexagona within the Bayou L'ourse sample. This population contains alternate alleles for each of the "diagnostic" loci as well as low frequency alleles found only in I. fulva or I. hexagona (tables 2 and 4). As with the Marmande sample there are also some low frequency alleles present in this population that are otherwise restricted to the $I$. brevicaulis sample. There are no known collections of $I$. brevicaulis from this region. It is conceivable, however, that such populations existed in the past. If this is the case, the allelic composition of the Bayou L'ourse and Marmande populations may reflect past introgression and the subsequent local extinction of $I$. brevicaulis. We have combined the rDNA and allozyme data from a transect through the Bayou L'ourse population to visualize patterns of genetic variation (fig. 1). Bennett (1989) demonstrated, from a two-year demographic analysis, that a significant component of the demography of the Bayou L'ourse population results from clonal reproduction. However, the previous molecular analysis (Arnold et al., 1990) and the present study indicate the presence of numerous hybrid genotypes. Furthermore, the isozyme analysis of Bayou L'ourse has determined that eight additional individuals have a hybrid phenotype (e.g., compare fig. 1 from this study with fig. 4 of Arnold et al., 1990). Therefore, sexual reproduction has apparently contributed significantly to the genetic structure of this hybrid population.

Molecular and biochemical data can also be used to indicate the presence or absence of putative parental, $F_{1}$ and advanced generation hybrids. The previous molecular analysis identified a number of individuals that possessed only the I. hexagona repeat length variant, but no individuals that contained only the I. fulva rDNA repeat length variant (Arnold et al., 1990). Likewise, the allozyme survey revealed several "I. hexagona" individuals, one putative $F_{1}$ individual that is heterozygous for each of the allozyme and rDNA markers, a number of plants that contained a predominance of either $I$. fulva or I. hexagona markers, but no individuals that were completely I. fulva (fig. 1). This supports the contention that Bayou L'ourse represents localized introgression between I. fulva and I. hexagona. Furthermore, Arnold et al. (1990) suggested that the I. fulva genotype might be undergoing genetic swamping due to the relatively smaller size of natural populations of this species, the superior competitive ability of $I$. hexagona (Bennett, 1989) and/or the loss of I. fulva habitat at the Bayou L'ourse site due to man-made disturbances (fig. 1).

The distribution of the genotypic variation in the Bayou L'ourse population does not appear to have a "clinal" (Huxley, 1938) pattern. Arnold et al. (1990) argued that, in contrast to the purely genetic prediction, the distribution of parental and 
hybrid genotypes may be directly related to the distribution of microhabitats within the site. Bennett (1989) and Bennett and Grace (1990) investigated the relative fitness of $I$. fulva, $I$. hexagona and hybrid generations under varying experimental conditions and found that $I$. fulva was the most shade tolerant of any of the classes examined. Hybrid individuals have intermediate shade tolerance, and I. hexagona was the most shade intolerant (Bennett and Grace, 1990). Differences in fitness relative to shading have been considered to be a major factor determining the geographic distribution of I. fulva, I. hexagona and their hybrids (Viosca, 1935), and the apparent habitat preferences of these species agree with the experimental findings. The competitive ability of the two species and the hybrid classes have also been shown to differ (Bennett, 1989). I. hexagona and the one hybrid class examined were equivalent in their competitive abilities, and both were superior to I. fulva. Water depth is another environmental variable that has been cited as a potential factor in the distribution of I. fulva, I. hexagona and the hybrid types. Differences in the relative fitness of the genotypes in the different microhabitats may also explain the absence of "pure" I. fulva individuals. Man-made disturbance would reduce shading and, thus, could shift the competitive balance towards individuals with $I$. hexagona genotypes.

It is also possible that detailed spatial analyses of genetic variation in the allopatric, introgressed populations (i.e., Marmande and Kraemer) may discern heterogeneity associated with ecological variation. Such a finding has been made with respect to the distribution of parental and hybrid types within a hybrid zone between the cricket species Gryllus pennsylvanicus and $G$. firmus (Rand and Harrison, 1989). In this example, Rand and Harrison (1989) found a strong correlation between the soil type of particular populations, the distribution of genetic and morphological markers and the degree of reproductive isolation.

\section{Introgressive hybridization in Louisiana irises:}

I. fulva $\times 1$. hexagona $\times I$. brevicaulis.

A mixture of allozyme markers diagnostic for $I$. fulva, I. hexagona and $I$. brevicaulis occurs in the Foti population (tables 3 and 4; fig. 2). This population occurs along the margin of Bayou Teche, borders on an area of unimproved pasture (fig. 2), and contains allozyme genotypes that are best explained by reciprocal crossing between each of the three species. This patern of genetic variation is suggestive of localized introgression between each of these species, however, the contribution of I. fulva, I. brevicaulis and I. hexagona to the genetic composition of this population is apparently unequal. Alleles characteristic of $I$. hexagona are found in only five individuals (fig. 2). In contrast, I. fulva and I. brevicaulis alleles are present in 35 and 40 of the 48 individuals, respectively (fig. 2). A preliminary analysis of ribosomal DNA from 1 . brevicaulis (Arnold, unpublished data) has identified a repeat length variant that is intermediate $(c a .11 \mathrm{~kb})$ between that found in I. fulva $(10 \mathrm{~kb})$ and I. hexagona $(13 \mathrm{~kb}$; Arnold et al., 1990). An analysis of rDNA variation in nine of the individuals from the Foti population has identified the presence of two repeat length variants approximately 10 and $11 \mathrm{~kb}$ in length (Arnold, unpublished data). As in the Bayou L'ourse sample, the spatial genetic structure of the Foti population is not strongly clinal and may be the result of habitat selection.

\section{Hybrid speciation in Louisiana irises: \\ I. nelsonii.}

Randolph (1966) and Randolph et al. (1967) argued that $I$. nelsonii represents a stabilized hybrid derivative of I. fulva, I. hexagona and possibly I. brevicaulis. The reproductive isolation between $I$. nelsonii and the other three species and the genetic stability of $I$. nelsonii has not been documented experimentally. However, populations of $I$. nelsonii are highly fertile as measured by pollen viability although they are apparently endemic to a region of southern Louisiana in which I. brevicaulis, I. fulva and I. hexagona occur and hybridize (Randolph et al., 1967). I. nelsonii has been reported to occupy a unique habitat relative to the other three species; populations of I. nelsonit were found in areas of relatively heavy shade and deep water (Randolph et al., 1967). Randolph et al. (1967) concluded that $I$. nelsonii possessed a combination of morphological characteristics that were found in I. fulva and I. hexagona. Furthermore, the shape of the seed capsule and later flowering time of $I$. nelsonii led Randolph (1966) to suggest a genetic contribution from I. brevicaulis as well. Chromosomal markers were also identified for I. nelsonii and were compared to the unique marker chromosomes of I. fulva, I. hexagona and I. brevicaulis (Randolph et al., 1961). Their analysis indicated that $I$. nelsonii possessed at least one of the marker chromosomes characteristic of I. fulva and a marker chromosome similar to one found in $I$. hexagona, but with a different centromere 
position (Randolph et al., 1961). None of the marker chromosomes from I. brevicaulis were present in the $I$. nelsonii karyotype.

If $I$. nelsonii is a hybrid derivative, it should possess alleles that characterize the putative parental taxa (i.e., I. fulva, I. hexagona and/or I. brevicaulis). Furthermore, the case for hybrid origin of $I$. nelsonii would be strengthened if some or all of the allozyme markers were also present in contemporary hybrid populations.

Allele frequencies present in I. nelsonii largely reflect those of $I$. fulva. This is apparent from the relatively high genetic identity between these two species $(I=0.95)$. However, as predicted by the hybrid derivative hypothesis, $I$. nelsonii also shares alleles with $I$. hexagona and $I$. brevicaulis (table 4). A comparison of the alleles present in the Foti population with those found in $I$. nelsonii further supports the contention that $I$. nelsonii is a derivative of introgressive hybridization between these three species.

The genetic stability of $I$. nelsonii is reflected by the high level of fertility of individual plants (Randolph et al., 1967). Grant (1981) states that an integral part of hybrid speciation is the stabilization of the "breeding behavior" of hybrid individuals. Grant (1981) further suggested several mechanisms that may act to reduce the disruption of hybrid gene complexes by recombination and segregation. These mechanisms included asexual reproduction (apomixis), permanent translocation heterozygosity, permanent odd polyploidy, allopolyploidy, recombinational speciation (i.e., the product of a hybrid derivative that is isolated from the parental species by diploid, chromosomal barriers) and the production of a hybrid type that is isolated by "external barriers". A combination of the above mechanisms may have played a role in the stabilization of $I$. nelsonii. First, clonal spread (due to a rhizomatous habit) is a significant aspect of the life-history of all of the Iris species examined in this study (Bennett, 1989). Therefore, the spread of the I. nelsonii hybrid derivative could have been facilitated by clonal reproduction following hybridization. Second, since this species apparently occupies a habitat that is unique in comparison to I. fulva, I. hexagona and $I$. brevicaulis (i.e., heavily shaded areas with deep water), an ecological separation between $I$. nelsonii and the parental species may also have assisted in its stabilization. Finally, I. hexagona, I. fulva, I. brevicaulis and $I$. nelsonii each are characterized by unique marker chromosomes (Randolph et al., 1961) indicating that $I$. nelsonii may be a hybrid derivative that is stable with regard to a "hybrid" chromosomal complement. Multiple avenues for the stabilization of hybrid derivatives should enhance the likelihood of hybrid speciation and would be possible whenever there is any combination of asexual and sexual reproduction, chromosomal differentiation and available ecological niches for hybrid derivatives to invade.

Acknowledgements We thank S. L. Sherman-Broyles for providing excellent technical assistance during the entire electrophoretic analysis. We are grateful to B. Marmande, K. Granier, L. Talbot, A. J. Butaud and F. Foti for permission to collect Iris samples on their property and to F. Givens and T. Hebert for invaluable assistance in locating and identifying a number of the populations. This research was supported by a grant from the American Iris Society Foundation (B.D.B. and M.L.A.) and NSF grant BSR-8718803 (J.L.H.).

\section{REFERENCES}

ANDERSON, E. 1949. Introgressive Hybridization. John Wiley \& Sons, Inc., New York.

ANDERSON, E. AND HUBRICHT, L. 1938. Hybridization in Tradescantia. III. The evidence for introgressive hybridization. Amer. J. Bot. 25, 396-402.

ARNOLD, M. L., BENNETT, B. D. AND ZIMMER, E. A. 1990. Natural hybridization between Iris fulva and I. hexagona: pattern of ribosomal DNA variation. Evolution, (in press).

ARNOLD, M. L., CONTRERAS, N. AND SHAW, D. D. 1988. Biased gene conversion and asymmetrical introgression between subspecies. Chromosoma, 96, 368-371.

ARNOLD, M. L., SHAW, D. D. AN D CONTRERAS, N. 1987. Ribosomal RNA-encoding DNA introgression across a narrow hybrid zone between two subspecies of grasshopper. Proc. Natl Acad. Sci. USA, 84, 3946-3950.

BAKER, R. J., DAVIS, S. K., BRADLEY, R. D., HAMILTON, M. J. AND VAN DEN BUSSCHE, R. A. 1989. Ribosomal-DNA, mitochondrial-DNA, chromosomal, and allozymic studies on a contact zone in the pocket gopher, Geomys. Evolution, $43,63-75$.

BENNETT, B. D. 1989. Habitat differentiation of Iris fulva Ker Gawler, Iris hexagona Walter, and their hybrids. Ph.D. Thesis, Louisiana State University.

BENNETT, B. D. AND GRACE, J. B. 1990. Shade tolerance and its effect on the segregation of two species of Louisiana Iris and their hybrids. Amer. J. Bot., 77, 100-107.

BERT, T. M. AND HARRISON, R. G. 1988. Hybridization in western Atlantic stone crabs (Genus Menippe): evolutionary history and ecological context influence species interactions. Evolution, 42, 528-544.

DEPAMPHILIS, C. W. AND WYATT, R. 1990. Electrophoretic confirmation of interspecific hybridization in Aesculus (Hippocastanaceae) and the genetic structure of a broad hybrid zone. Evolution, (in press).

DOYLE, J. J. AND DOYLE, J. L. 1988. Natural hybridization in eastern North American Claytonia. Amer. J. Bot., 75, 1238 1246.

GOTTLiEB, L. D. 1981. Electrophoretic evidence and plant populations. Progr. Phytochem., 7, 1-46.

GRANT, v. 1981. Plant Speciation. Columbia University Press, New York

HARDIN, J. W. $1957 a$. Studies in the Hippocastanaceae, III. A hybrid swarm in the buckeyes. Rhodora, 59, 45-51. 
HARDIN, J. W. 1957b. Studies in the Hippocastanaceae, IV. Hybridization in Aesculus. Rhodora, 59, 185-203.

HARRISON, R. G. 1986. Pattern and process in a narrow hybrid zone. Heredity, 56, 337-349.

HEISER, C. B. 1949. Study in the evolution of the sunflower species Helianthus annuus and $H$. bolanderi. Univ. Calif. Publ. Bot., 23, 157-196.

HEISER, C. B., SMITH, D. M., CLEVENGER, S. B. AND MARTIN, W. C. 1969. The North American sunflowers (Helianthus). Mem. Torrey Botan. Club, 22, 1-218.

HUXLEY, J. 1938. Clines: an auxiliary taxonomic principle. Nature, 142, 219-220.

MARCHANT, A. D., ARNOLD, M. L. AND WILKINSON, P. 1988. Gene flow across a chromosomal tension zone. I. Relicts of ancient hybridization. Heredity, 61, 321-328.

MITTON, J. B., LINHART, Y. B., STURGEON, K. B. AND HAMRICK, J. L. 1979. Allozyme polymorphisms detected in mature needle tissue of ponderosa pine. J. Heredity, 70 , $86-89$.

MORAN, C. AND SHAW, D. D. 1977. Population cytogenetics of the genus Caledia (Orthoptera: Acridinae). III. Chromosomal polymorphism, racial parapatry and introgression. Chromosoma, 63, 181-204.

NEI, M. 1972. Genetic distance between populations. Amer. Nat., 106, 283-292.

POTTS, B. M. AND REID, J. B. 1988. Hybridization as a dispersal mechanism. Evolution, 42, 1245-1255.

RAND, D. M. AND HARRISON, R. G. 1989. Ecological genetics of a mosaic hybrid zone: mitochondrial, nuclear, and reproductive differentiation of crickets by soil type. Evolution, 43, 432-449.

RANDOLPH, L. F. 1966. Iris nelsonii, a new species of Louisiana iris of hybrid origin. Baileya, 14, 143-169.
RANDOLPH, L. F., MITRA, J. AND NELSON, I. S. 1961. Cytotaxonomic studies of Louisiana irises. Bot. Gaz, 123, 126-133.

RANDOLPH, L. F., NELSON, I. S. AND PLAISTED, R. L. 1967. Negative evidence of introgression affecting the stability of Louisiana Iris species. Cornell Univ. Agr. Expt. Stat. Memoir, 398, 1-56.

RIESEBERG, L. H., CARTER, R. AND zONA, S. 1990. Molecular tests of the hypothesized hybrid origin of two diploid Helianthus species (Asteraceae). Evolution, (in press).

RIESEBERG, L. H., SOLTIS, D. E. AND PALMER, J. D. 1988. A molecular reexamination of introgression between Helianthus annuus and $H$. bolanderi (Compositae). Evolution, 42, 227-238.

RILEY, H. P. 1938. A character analysis of colonies of Iris fulva, Iris hexagona var. giganticaerulea and natural hybrids. Amer. J. Bot., 25, 727-738.

SHAW, D. D., MORAN, C. AND WILKINSON, P. 1980. Chromosomal reorganization, geographic differentiation and the mechanism of speciation in the genus Caledia. In Blackman, R. L., Hewitt, G. M. and Ashburner, M. (eds) Insect Cytogenet., Symp. R. Entomol. Soc. Lond., no. 10, pp. 171194.

SOLTIS, D. E., HAUFLER, C. H., DARROW, D. C. AND GASTONY, G. J. 1983. Starch gel eletrophoresis of ferns: a compilation of grinding buffers, gel and electrode buffers, and staining schedules. Amer. Fern J., 73, 9-27.

SOLTIS, P. S. 1985. Studies of genetic variation in an introgressive complex in Clarkia (Onagraceae). PhD. Thesis, University of Kansas.

VIOSCA, P., JR. 1935. The irises of southeastern Louisiana: a taxonomic and ecological interpretation. J. Amer. Iris. Soc., 57, 3-56. 